\title{
Investigating the observational signatures of magnetic cloud substructure
}

\author{
K. Steed, ${ }^{1}$ C. J. Owen, ${ }^{1}$ P. Démoulin, ${ }^{2}$ and S. Dasso ${ }^{3}$ \\ Received 16 July 2010; revised 6 September 2010; accepted 12 October 2010; published 26 January 2011.
}

[1] Magnetic clouds (MCs) represent a subset of interplanetary coronal mass ejections

(ICMEs) that exhibit a magnetic flux rope structure. They are primarily identified by smooth, large-scale rotations of the magnetic field. However, both small- and large-scale fluctuations of the magnetic field are observed within some magnetic clouds. We analyzed the magnetic field in the frames of the flux ropes, approximated using a minimum variance analysis (MVA), and have identified a small number of MCs within which multiple reversals of the gradient of the azimuthal magnetic field are observed. We herein use the term "substructure" to refer to regions that exhibit this signature. We examine, in detail, one such MC observed on 13 April 2006 by the ACE and WIND spacecraft and show that substructure has distinct signatures in both the magnetic field and plasma observations. We identify two thin current sheets within the substructure and find that they bound the region in which the observations deviate most significantly from those typically expected in MCs. The majority of these clouds are followed by fast solar wind streams, and a comparison of the properties of this magnetic cloud with five similar events reveals that they have lower nondimensional expansion rates than nonovertaken magnetic clouds. We discuss and evaluate several possible explanations for this type of substructure, including the presence of multiple flux ropes and warping of the MC structure, but we conclude that none of these scenarios is able to fully explain all of the aspects of the substructure observations.

Citation: Steed, K., C. J. Owen, P. Démoulin, and S. Dasso (2011), Investigating the observational signatures of magnetic cloud substructure, J. Geophys. Res., 116, A01106, doi:10.1029/2010JA015940.

\section{Introduction}

[2] Magnetic clouds (MCs) are a subset of interplanetary coronal mass ejections (ICMEs), typically identified, at least initially, by three main criteria: the magnetic field rotates smoothly over a large angle during an interval of the order of 1 day, the magnetic field strength is higher than in the typical solar wind, and the proton temperature is lower than in the typical solar wind [Burlaga et al., 1981; Klein and Burlaga, 1982; Burlaga, 1995].

[3] Magnetic clouds have been widely modelled as largescale, force-free, cylindrical magnetic flux ropes [e.g., Lepping et al., 1990; Marubashi, 1997; Lynch et al., 2003; Dasso et al., 2005]. In this scenario, the magnetic field is helical, such that it is in the axial direction toward the center of the flux rope and in the azimuthal direction at the flux rope boundary. As a magnetic cloud passes over a spacecraft located in the solar wind, a bipolar signature, observed in the

\footnotetext{
${ }^{1}$ Mullard Space Science Laboratory, University College London, Dorking, Surrey, UK.

${ }^{2}$ Observatoire de Paris, Meudon, Paris, France.

${ }^{3}$ Departmento de Fisica and Instituto de Astronomia y Fisica del Espacio, Universidad de Buenos Aires, Buenos Aires, Argentina.

Copyright 2011 by the American Geophysical Union. 0148-0227/11/2010JA015940
}

azimuthal magnetic field component, is anticipated. This is indicative of the large-scale rotation of the magnetic field within the structure. However, this rotation is not always smooth and often exhibits both small- and large-scale fluctuations of the magnetic field within the cloud [Crooker et al., 1990]. Various physical processes have been proposed that may influence the topology of a magnetic cloud, both near to the Sun and further out in the heliosphere, including magnetic reconnection [Gosling et al., 2007], interaction between multiple magnetic flux ropes [e.g., Fainberg et al., 1996; Lepping et al., 1997; Osherovich et al., 1999; Farrugia, 2001], spontaneous formation of current sheets [Owens, 2009], or interaction with dust trails originating from comets [Russell et al., 2009].

[4] In a small handful of magnetic clouds, multiple reversals of the main gradient of the azimuthal magnetic field component are observed toward the center of the cloud [Dasso et al., 2007]. We herein use the term "substructure" to refer only to the region of a magnetic cloud that exhibits this signature. Dasso et al. [2007] suggest that in a magnetic flux rope structure, the magnetic flux surfaces of an MC could become "warped" as a result of its fast evolution and interaction with the ambient solar wind, giving rise to the observed magnetic field fluctuations. However, this and other possible causes of this kind of substructure have not, as yet, been investigated further. 
[5] In this study, we discuss the characteristics and observational signatures associated with the presence of substructure toward the center of some magnetic clouds. In section 2, we describe, in detail, the magnetic field and plasma observations of the substructure present within a magnetic cloud observed by the Advanced Composition Explorer (ACE) and WIND spacecraft on 13 April 2006. In section 3 , we investigate the prevalence of substructure within magnetic clouds, examining and comparing the properties of the clouds exhibiting substructure that we have identified, and in section 4, we discuss and evaluate some possible scenarios that might explain the presence of substructure in these magnetic clouds.

\section{In Situ Observations of the 13 April 2006 Magnetic Cloud}

\subsection{Overview of the Event}

[6] Figure 1 shows data taken in the magnetic cloud encountered by the ACE and WIND spacecraft on 13 April 2006. This MC displays many of the typical characteristics used to identify a magnetic cloud [Steed et al., 2008] and also exhibits the clearest example of substructure toward its center that we have found. Magnetic field data from the Magnetic Field Investigation [MFI, Lepping et al., 1995] and plasma data from the 3-D Plasma and Energetic Particle Experiment [3DP, Lin et al., 1995] on the WIND spacecraft, located upstream of Earth, are shown at $3 \mathrm{~s}$ resolution. Figures 1a and $1 \mathrm{~b}$ show the total and $B_{x}, B_{y}$, and $B_{z}$ components of the magnetic field in Geocentric Solar Ecliptic (GSE) coordinates, respectively. Figure 1c shows the bulk flow speed of the protons, Figure 1d shows the proton number density, and Figure 1e shows the radial proton temperature. Figure 1f shows the pitch angle velocity distributions of suprathermal electrons at $272 \mathrm{eV}$ observed by the Solar Wind Electron Proton Alpha Monitor/STEA [SWEPAM, McComas et al., 1998] on the ACE spacecraft, which has been temporally aligned with observations of the substructure region at WIND (see section 2.4). Counterstreaming beams of suprathermal electrons are typically associated with ICMEs [Zwickl et al., 1983; Gosling et al., 1987] and tell us about the connectivity of magnetic field lines to the Sun. When a counterstreaming beam of suprathermal electrons is observed, it indicates the presence of closed field lines that are still connected to the Sun at both ends. It is common for unidirectional beams to also be observed within an ICME, indicating that one of the foot points is no longer connected to its solar source, while complete dropouts in the observations of these electrons indicates that the magnetic structure has become completely disconnected from the Sun. Figure 1f shows that counterstreaming suprathermal electron beams at $\sim 272 \mathrm{eV}$ are observed throughout much of this magnetic cloud, indicating that the ejecta is still connected to the Sun when it is observed at ACE. However, the onset of these bidirectional beams is $\sim 1 \mathrm{~h}$ prior to the onset of the magnetic cloud, as determined from the magnetic field observations, suggesting the presence of closed magnetic field lines propagating ahead of the magnetic cloud. We suggest that this may indicate that overlying coronal loops were also pushed out and ejected when this magnetic cloud erupted. We also note that the observations show that the beam is unidirectional as the spacecraft emerges from the substructure region and moves into the back part of the magnetic cloud, indicating that the magnetic structure is partially disconnected from the Sun at one of its foot points in this region.

[7] At the boundary of a magnetic flux rope, a current sheet is generally expected since there is a change of connectivity of magnetic field lines (from twisted field lines inside the flux rope to solar wind-like field lines outside). Then, the in- and out-bound boundaries in in situ data are typically expected where magnetic discontinuities are present [e.g., Dasso et al., 2006]. Large-scale, force-free, cylindrical models of magnetic flux ropes, like that of Lundquist [1950], suggest that the magnetic field in the axial direction is strongest toward the center of the flux rope, while the azimuthal magnetic field direction displays a large-scale rotation. Observationally, it is usual to see a peak in the axial magnetic field near the center of a magnetic cloud and it is expected that this component will tend to zero toward the boundaries of the magnetic cloud. A bipolar signature is often observed due to the reversal of the azimuthal magnetic field component of the flux rope, passing through zero toward the center of the magnetic cloud. All of these characteristics are guidelines to define the flux rope boundaries from the measured field components in the GSE coordinate system. In the 13 April $2006 \mathrm{MC}, B_{z}$ is dominated by the axial field component, while $B_{x}$ and $B_{y}$ have a dominant contribution from the azimuthal component (Figure 1b). However, these boundaries need to be confirmed after rotating the magnetic data in the local MC coordinate system in order to fully separate the azimuthal and axial components. We confirm the boundaries shown in Figure 1 by using a minimum variance analysis (MVA) (see section 2.2) to rotate the data to the MC frame, selecting the interval that shows the clearest rotation (Figure 2). The MV analysis gives a ratio between the intermediate and the minimum eigenvalues of $\sim 12$, which implies that the minimum variance direction is well defined [Siscoe and Suey, 1972]. The MC axis orientation was also determined using MVA by Steed et al. [2008] and is described by $\theta$ and $\phi$, where $\theta$ is the angle between the ecliptic plane and the MC axis and $\phi$ is the angle between $x_{\mathrm{GSE}}$ and the projection of the $\mathrm{MC}$ axis on the ecliptic plane (measured positive when anticlockwise). They found that $\theta \sim 68^{\circ}$ and $\phi \sim 294^{\circ}$.

[8] Vertical dot-dashed black lines indicate the boundaries of the magnetic cloud at WIND, which are found to be very similar to those determined by Steed et al. [2008], using data from the ACE spacecraft. Unusual magnetic field and plasma observations persist beyond the identified rear boundary of this magnetic cloud and we suggest that this may be the result of interaction between the magnetic cloud flux rope and the solar wind. Dasso et al. [2006] reported that magnetic reconnection can be forced in front of an $\mathrm{MC}$ when a flux rope is overtaking the magnetic field ahead of it. The consequence of this reconnection is a flux tube that is peeled away at the front but has an extended back part. This "back" region is expected to exhibit different behavior to both the main body of the ejecta and the solar wind. Steed et al. [2008] investigated several locations for the rear boundary of this MC and, despite the complexity of the observations toward the rear of the ejecta, they found that changes in the rear boundary location over a time interval spanning almost $10 \mathrm{~h}$ did not result in major changes in 


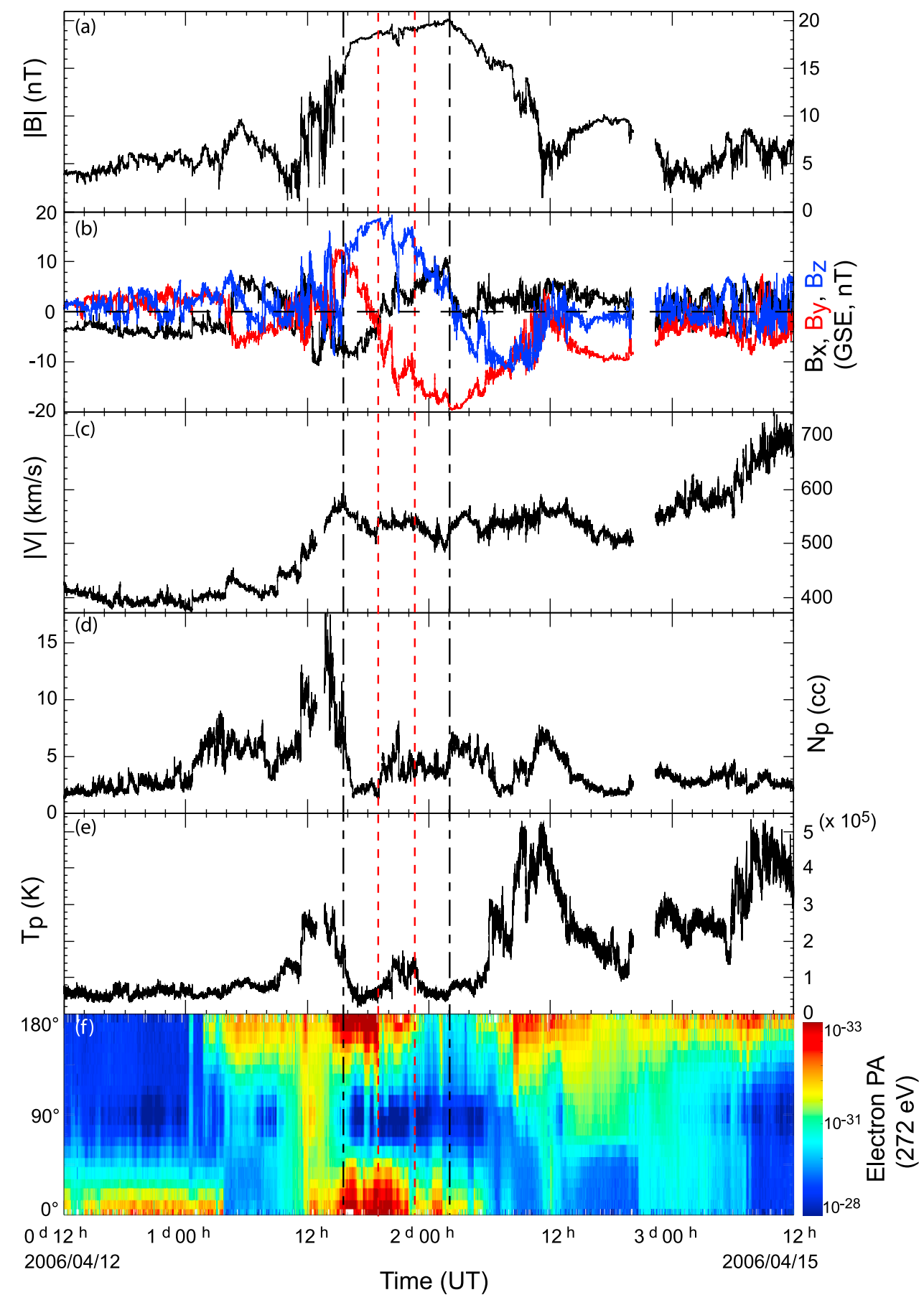

Figure 1. Interplanetary magnetic field and plasma data from the WIND spacecraft during the passage of the ICME ejecta in GSE coordinates. Vertical dot-dashed black lines indicate the boundaries of the magnetic cloud, and vertical dashed red lines indicate the boundaries of the substructure region. The following are shown: (a) the total and (b) the $B_{x}, B_{y}$, and $B_{z}$ components of the magnetic field, (c) proton bulk flow speed, (d) proton number density, (e) radial proton temperature, and (f) the pitch angle velocity distributions of the suprathermal electrons at $272 \mathrm{eV}$ (observed by ACE and temporally aligned with observations of the substructure region observed by WIND, see Figure 4).

the orientation of the MC axis. Within this magnetic cloud the rotation of the magnetic field evident in $B_{y}$ is not smooth, and vertical dashed red lines bound the region where the $B_{y}$ magnetic field observations deviate from the larger scale trend bipolar signature, exhibiting multiple reversals of the gradient of this magnetic field component toward the center of the cloud. Associated changes in the other magnetic field directions and the plasma measurements are also observed in this region. 


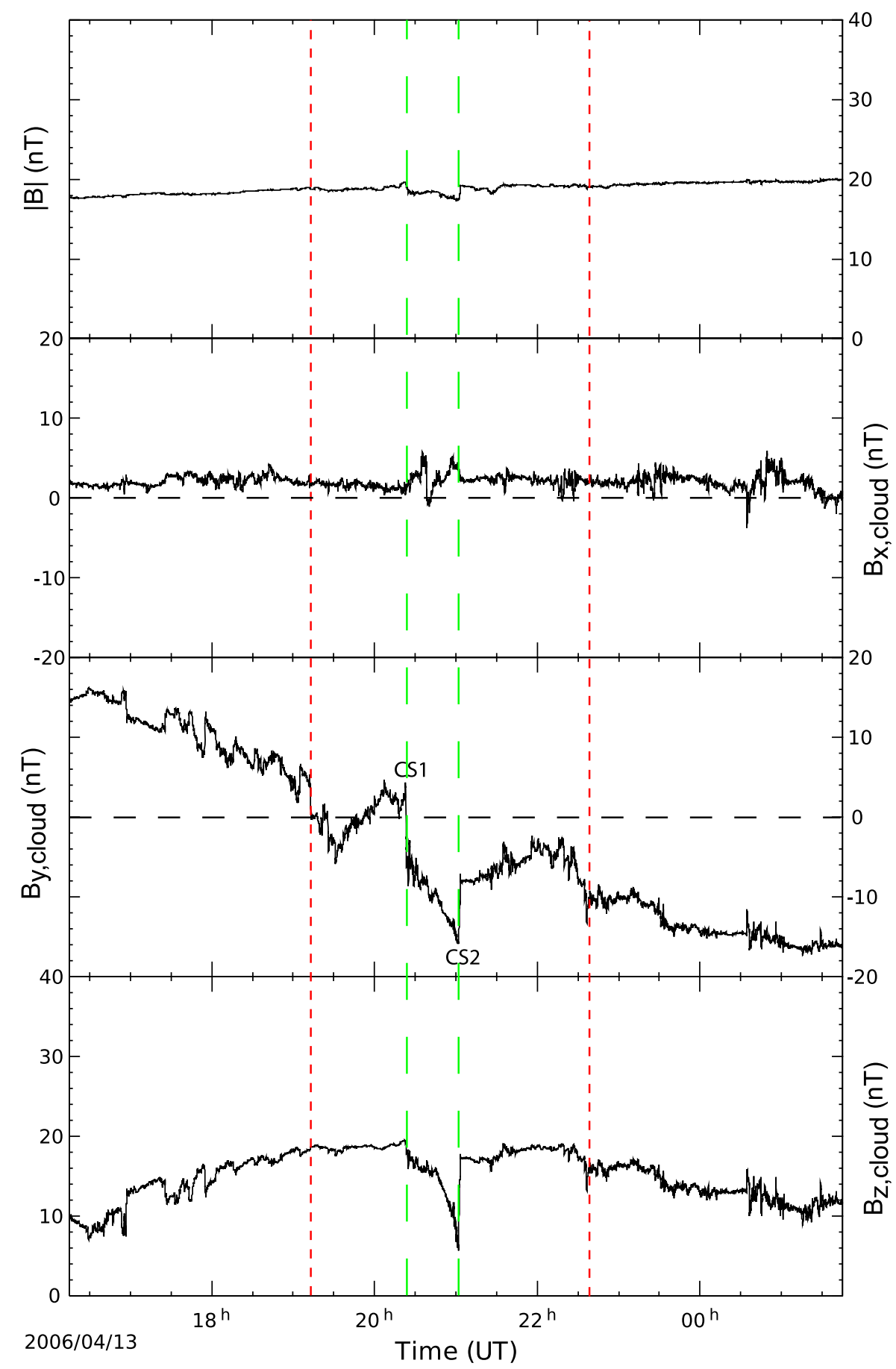

Figure 2. Magnetic field components in local magnetic coordinates obtained from a minimum variance analysis performed over the duration of the 13 April 2006 magnetic cloud. The following are shown: the magnitude of the magnetic field and the evolution of the magnetic field components in the minimum $\left(B_{x, \text { cloud }}\right)$, maximum $\left(B_{y, \text { cloud }}\right)$ and intermediate $\left(B_{z, \text { cloud }}\right)$ variance directions. Vertical short-dashed red lines indicate the boundaries of the substructure region, within which reversals of the azimuthal magnetic field $\left(B_{y, \text { cloud }}\right)$ gradient are observed. Discontinuities consistent with the presence of thin current sheets are also observed at 2023 UT (CS1) and 2102 UT (CS2), indicated by the vertical long-dashed green lines, coincident with an observed decrease in the axial magnetic field $\left(B_{z, \text { cloud }}\right)$ component and its subsequent recovery.

\subsection{Magnetic Field Observations}

[9] Figure 2 shows 3 s resolution data from the MFI instrument on the WIND spacecraft over the interval of the magnetic cloud, rotated to a local coordinate system defined using a minimum variance analysis (MVA) [Sonnerup and Cahill, 1967] on the magnetic field. This method can be used to find the natural coordinate system of a flux rope, allowing us to better see the nature of the fluctuations in the 
azimuthal and axial magnetic fields of the flux rope. The maximum variance $\left(B_{y, \text { cloud }}\right)$ direction contains the effects of the rotation of the azimuthal magnetic field and shows that the large-scale rotation over the duration of the 13 April 2006 magnetic cloud is positive to negative. However, a number of reversals of the gradient of this magnetic field component are observed between 1903 UT and 2238 UT on 13 April 2006. We have identified these signatures as substructure within the magnetic cloud. The boundaries of this substructure are again highlighted by vertical short-dashed red lines. Within the substructure region, relatively sharp discontinuities are observed at 2023 UT and 2102 UT, indicating the presence of thin current sheets, labeled CS1 and CS2 respectively and highlighted by vertical long-dashed green lines. The unusual substructure signatures observed in the $B_{y, \text { cloud }}$ magnetic field component of this magnetic cloud persisted for approximately $3.5 \mathrm{~h}$.

[10] The axial magnetic field is contained in the intermediate direction $\left(B_{z \text {,cloud }}\right)$ in our local coordinate system. It also departs from what is expected from a force-free, cylindrical flux rope model. Observations of $B_{z \text {,cloud }}$ show that toward the center of the $\mathrm{MC}$, where it is expected that the magnetic field is strongest, the magnetic field begins to decrease where $B_{z, \text { cloud }}$ has a discontinuity at 2023 UT, coinciding with the time at which we observe CS1. The decrease of $B_{z \text {,cloud }}$ is large, since it changes from 19 to $6 \mathrm{nT}$ between 2023 UT and 2102 UT. At 2102 UT when CS2 is observed, a sharp discontinuity is also observed in $B_{z \text {,cloud }}$, resulting in the rapid recovery of this magnetic field component to pre-disturbance levels. It is interesting to note that the onset of the substructure signatures in the azimuthal magnetic field occurs approximately $70 \mathrm{~min}$ before there are any notable changes observed in the axial magnetic field and that the observed reduction in the $B_{z \text {,cloud }}$ component of the magnetic field is mostly confined between CS1 and CS2. The observed large fluctuations in the axial and azimuthal components of the magnetic field are weakly reflected in the magnetic field magnitude, since there are only some small changes observed during this period of time when the surrounding magnetic field is very smooth.

\subsection{Corresponding Plasma Observations}

[11] The changes in the magnetic cloud, evident in the magnetic field observations, are accompanied by associated changes in the physical properties of the plasma in this region. Figure 3 shows 3 s resolution plasma measurements from the 3DP instrument on the WIND spacecraft, in GSE coordinates. Figures $3 \mathrm{a}$ and $3 \mathrm{~b}$ show translational proton velocity, $V_{x}$, and proton density, $N_{p}$, respectively. The expected temperature, $T_{e x}$, is typical of the plasma proton temperature found in the ambient solar wind and is determined by an empirical correlation with the observed solar wind speed, $V_{s w}$ [Lopez, 1987, and references therein]. Figure $3 \mathrm{c}$ shows the observed proton temperature, $T_{p}$ (black trace), and the expected temperature, $T_{\text {ex }}$ (red trace), calculated using the relation from Neugebauer et al. [2003], based on 3 years of measurements from ACE. We also show $T_{\text {ex }} / 2$ (blue trace). Figure $3 \mathrm{~d}$ shows the proton beta, Figures $3 \mathrm{e}$ shows the ratio of the relative abundances of helium to hydrogen, $N_{\alpha} / N_{p}$, and Figure $3 \mathrm{f}$ shows the magnitude, $B_{x}, B_{y}$, and $B_{z}$ components of the magnetic field in GSE coordinates, for ease of comparison with the plasma observations. The boundaries of the substructure region and the locations of the current sheets, as determined from the magnetic field observations, are highlighted by vertical short-dashed red lines and vertical long-dashed green lines, respectively.

[12] Observations of the translational proton velocity, $V_{x}$ in GSE coordinates, within the magnetic cloud (Figure 3a) show that the change in speed of the cloud as it passes over the WIND spacecraft, determined from observations of the translational velocity at its leading and trailing edges, is small $\left(\sim 40 \mathrm{~km} \mathrm{~s}^{-1}\right)$. The change in the translational velocity over the duration of an $\mathrm{MC}$ can be used to calculate its expansion rate. However, the difference, $\Delta V_{x}$, between the leading and trailing edges is not a direct indicator of the expansion rate of a plasma element, since, in particular, $\Delta V_{x}$ depends on the size of the magnetic cloud so larger magnetic clouds have typically larger $\Delta V_{x}$ values. A better measure of the expansion rate of the magnetic cloud is given by the nondimensional expansion rate, $\zeta$, defined from theoretical considerations by Démoulin et al. [2008] and from data analysis by Gulisano et al. [2010],

$$
\zeta=\frac{\Delta V_{x}}{\Delta t} \frac{D}{V_{c}^{2}}
$$

where $\Delta t$ is the time difference between the observations of the in- and out-bound boundaries, $V_{c}$ is the velocity of the magnetic cloud center, and $D$ is the distance to the Sun. A simple interpretation of $\zeta$ is obtained when $\zeta$ is independent of the distance, $D$, to the Sun. Then the size, $S$, of the magnetic cloud is simply evolving with distance, $D$, as

$$
S=S_{0}\left(D / D_{0}\right)^{\zeta}
$$

where $S_{0}$ is the reference size at the distance $D_{0}$. More generally, $\zeta$ measures the proportion by which the magnetic cloud is changing its size with solar distance during the spacecraft crossing (independently of its size, $S_{0}$ ). We find that $\zeta=0.57$ for the 13 April 2006 magnetic cloud.

[13] Increases in both the proton density and temperature are observed within the substructure region. Extreme density decreases (to $\leq 1 \mathrm{~cm}^{-3}$ ) are typically observed in ICMEs [Richardson et al., 2000] and similarly for the same bulk flow speed, low proton temperatures relative to the ambient solar wind are expected [Gosling, 1990; Richardson and Cane, 1995; Mulligan et al., 1999] as a result of expansion of the ICME in the solar wind. Richardson and Cane [1995] showed that $T_{p}<T_{\mathrm{ex}} / 2$ can be used as a criterion for identifying ICME ejecta in interplanetary space and Figure 3c shows that while much of the 13 April 2006 magnetic cloud meets this criterion, $T_{p}$ significantly exceeds the threshold set by $T_{\mathrm{ex}} / 2$ within the substructure region and here tends toward temperatures expected in the ambient solar wind at this $V_{\text {sw. }}$.

[14] Magnetic pressure usually dominates within magnetic cloud structures, resulting in low values of plasma $\beta$. Figure $3 \mathrm{~d}$ shows that this is also the case for this magnetic cloud, with a value of 0.01 calculated for proton $\beta$ in the main body of the cloud. However, between CS1 and CS2 the magnetic pressure undergoes a small decrease while the plasma pressure undergoes a small increase, leading to slightly elevated proton $\beta$ values of 0.08 in this region, higher than the value of 0.03 calculated in the surrounding substructure region. 


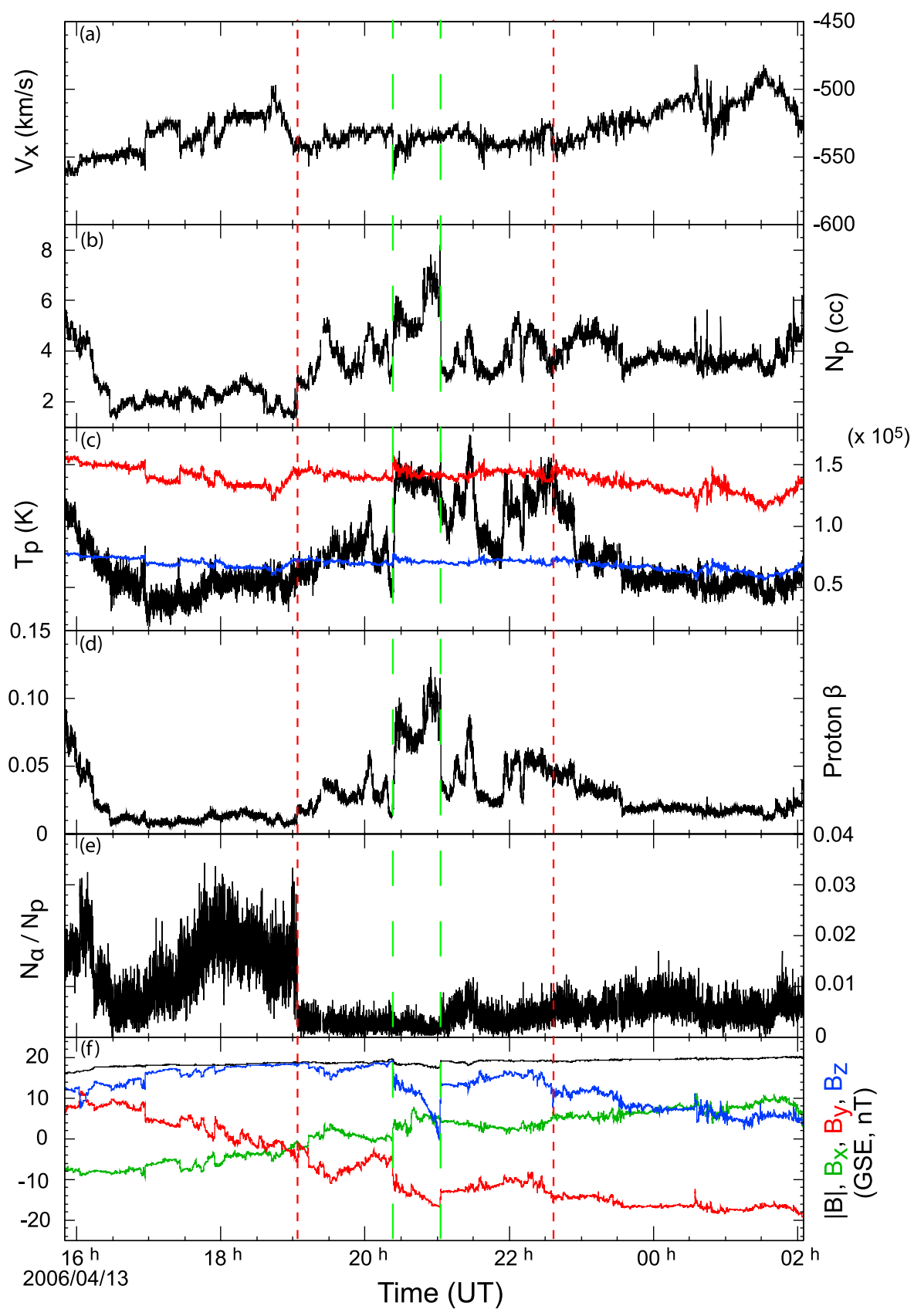

Figure 3. Plasma observations from WIND over the duration of the 13 April 2006 magnetic cloud. The following are shown (a) the translational proton velocity, $V_{x}$, in GSE coordinates, (b) the proton number density, (c) the radial proton temperature (black trace), the expected temperature, $T_{\text {ex }}$ (red trace), calculated from the observed solar wind speed and $T_{\mathrm{ex}} / 2$ (blue trace), (d) the proton $\beta$, (e) the ratio of the relative abundances of helium to hydrogen, and (f) the magnetic field observations in GSE coordinates, provided for context. Vertical short-dashed red lines indicate the boundaries of the substructure region, and vertical long-dashed green lines indicate the locations of the currents sheets (CS1 and CS2).

Despite this, these values are still very low relative to the value of 0.2 obtained in the surrounding solar wind.

[15] Elevated $\alpha$-particle (ionized helium) abundances are often observed in solar ejecta [Hirshberg et al., 1972; Zwickl et al., 1983; Mulligan et al., 1999; Richardson and Cane, 2004]. $N_{\alpha} / N_{p}$, the ratio of the relative abundances of helium to hydrogen, is typically $\sim 8 \%$ in ICMEs, compared to a value nearer to $3-5 \%$ in the ambient solar wind. Figure $3 \mathrm{e}$ shows that the abundance of helium within the presubstructure part of the cloud is lower than is typically observed within an ICME, approaching 3\%, but this is still slightly higher than the value of $2 \%$ observed in the surrounding solar wind. At 1903 UT, corresponding with the onset of the substructure region, the abundance of helium decreases 


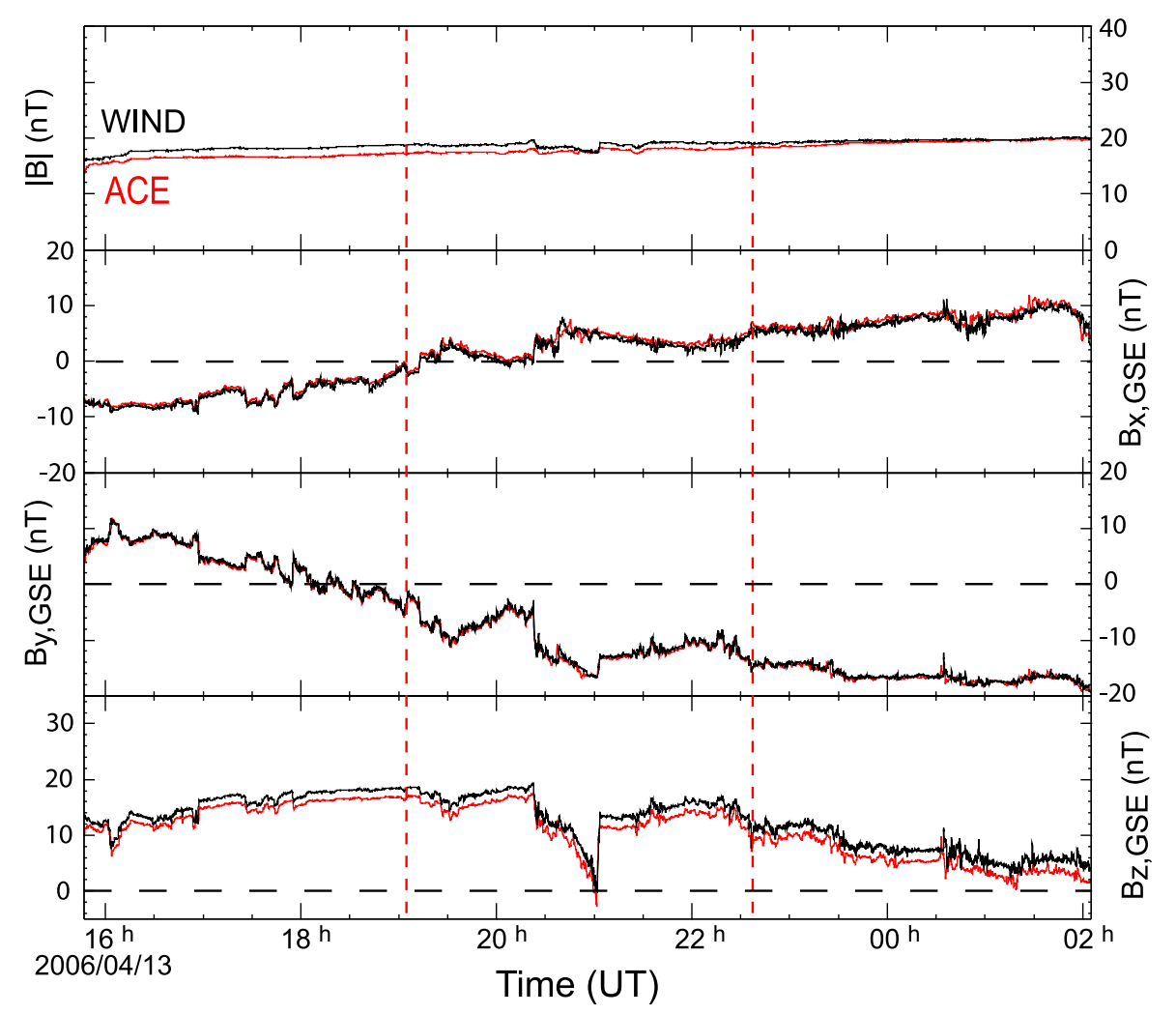

Figure 4. Magnetic field observations in GSE coordinates over the duration of the 13 April 2006 magnetic cloud by WIND (black trace) and ACE (red trace). Vertical dashed red lines indicate the boundaries of the substructure region. The ACE data have been time shifted by $528 \mathrm{~s}(\sim 9 \mathrm{~min})$ so the WIND and ACE observations may be directly compared. This shows that the observations of the magnetic structure from both spacecraft are very similar.

rapidly to a mean value of $0.3 \%$ and is sustained at this level throughout the substructure region. Notably, as the spacecraft moves through the substructure region and back into the main structure of the magnetic cloud the helium abundance remains very low.

\subsection{A Comparison of Multiple Spacecraft Observations}

[16] The 13 April 2006 magnetic cloud is observed by both the ACE and WIND spacecraft, allowing us to compare observations of this event, in particular its substructure, from two spacecraft. In GSE coordinates, the position of the ACE spacecraft is $x=240 R_{E}, y=25 R_{E}, z=-25 R_{E}$. At this time, the position of the WIND spacecraft is $x=200 R_{E}, y=$ $30 R_{E}, z=-15 R_{E}$. Both ACE and WIND were located close to the Sun-Earth line, with ACE observing the magnetic cloud first.

[17] Figure 4 shows magnetic field observations from ACE and WIND over the duration of the magnetic cloud. Cross-correlation is used to compare these data sets within the substructure region and returns a maximum correlation coefficient $>0.9$ when the WIND observations lag the ACE observations by approximately $528 \mathrm{~s}(\sim 9 \mathrm{~min})$. Therefore, in order to obtain a direct comparison of the magnetic cloud observations from each spacecraft, the ACE data has been time shifted by $528 \mathrm{~s}$. The boundaries of the substructure region are highlighted by vertical dashed red lines, as before. We find that the ACE and WIND observations of the magnetic field are very similar.

\section{Prevalence of Substructure Within Magnetic Clouds}

[18] We have surveyed all 48 of the magnetic clouds observed between March 2001 and November 2007, recorded in the WIND magnetic cloud catalog (http://lepmfi.gsfc.nasa. gov $/ \mathrm{mfi} / \mathrm{mag}$ _cloud_pub1.html). To ascertain what proportion of the magnetic clouds observed near Earth exhibit substructure, magnetic field observations of each MC from the MFI instrument onboard the WIND spacecraft were visually inspected for evidence of substructure, and a subset of magnetic clouds containing these features was identified. A minimum variance analysis was then performed on the magnetic field measurements of each of the clouds in this subset to further enhance the observational signatures of substructure by rotating the magnetic field vector to the eigenvector frame given by the minimum variance (an approximation of the magnetic cloud frame).

[19] We have found that in around $60 \%$ of these magnetic clouds, the magnetic field does not rotate entirely smoothly, often exhibiting both small- and large-scale fluctuations. However, we have identified only five magnetic clouds, in addition to the 13 April 2006 cloud, that exhibit the multiple 


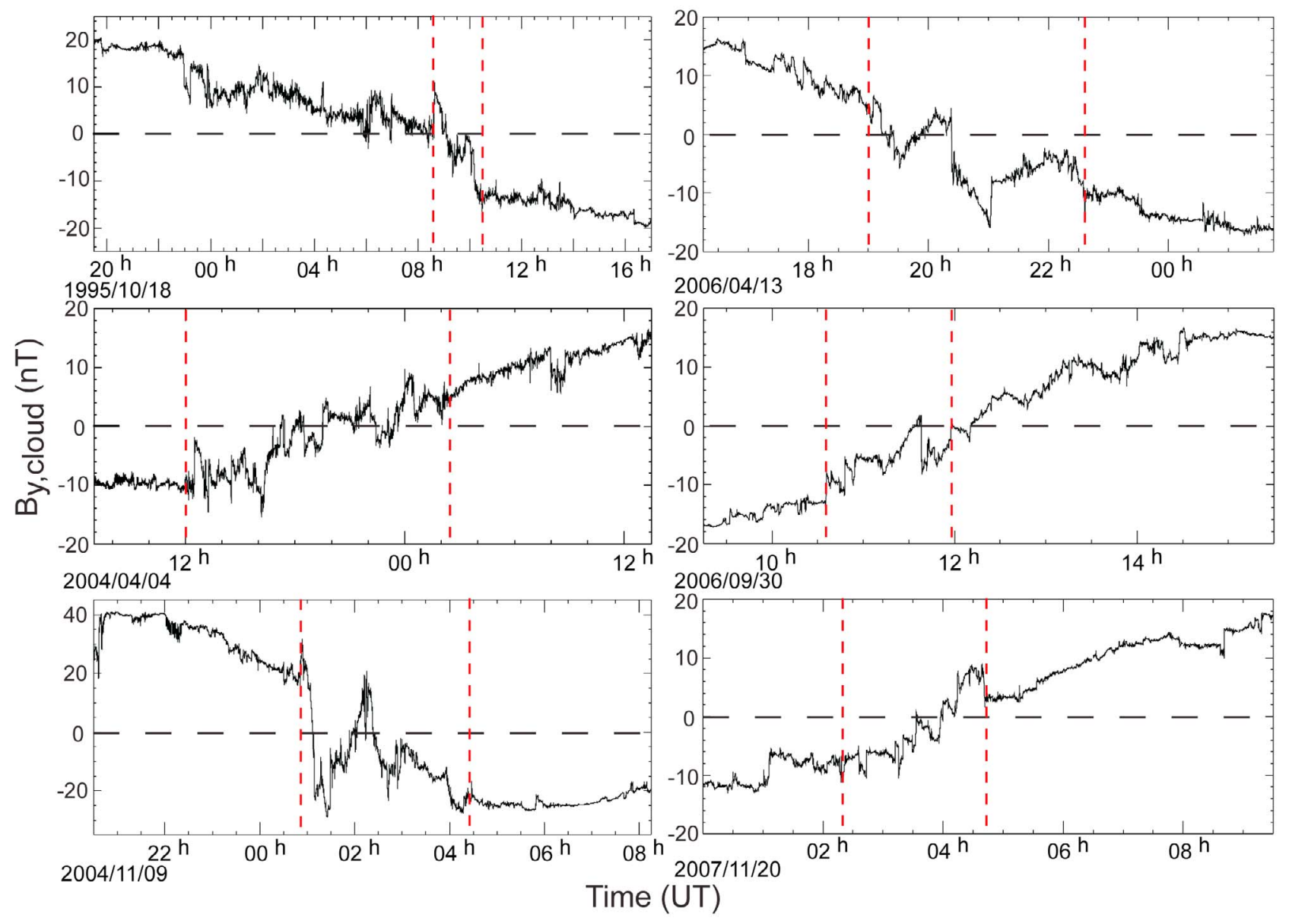

Figure 5. The evolution of the $B_{y, \text { cloud }}$ magnetic field component (maximum variance direction) is shown over the duration of all six of the magnetic clouds exhibiting substructure. Vertical dashed red lines indicate the boundaries of the substructure regions, within which reversals of the azimuthal magnetic field $\left(B_{y, \text { cloud }}\right)$ gradient are observed.

reversals in the gradient of the azimuthal magnetic field in which we are interested. For those magnetic clouds that have already been the subject of study by other researchers, we use the most recently discussed boundaries, while the remainder of the magnetic cloud boundaries are as stated in the WIND magnetic cloud catalog (estimated using the Lepping et al. [1990] magnetic field model). Figure 5 shows the evolution of the $B_{y \text {,cloud }}$ component of the magnetic field, corresponding to the maximum variance direction obtained from the minimum variance analysis, over the duration of all six substructure magnetic clouds identified. Vertical dashed red lines indicate the approximate boundaries of the substructure regions, within which reversals of the azimuthal magnetic field ( $\left.B_{y, \text { cloud }}\right)$ gradient are observed.

[20] Here, we compare some of the properties of these magnetic clouds, allowing us to build a general picture of the characteristics of these types of solar ejecta. We estimate the trajectories of the WIND spacecraft through this subset of magnetic clouds using a technique developed by Gulisano et al. [2007], which is valid for the force-free Lundquist [1950] model, and in which the magnetic field components obtained from MVA are used to provide an approximation of the impact parameter, $p$, defined as the minimum distance from the spacecraft to the cloud axis. The deviation from zero of the mean value of the $\mathrm{B}_{x \text {,cloud }}$ component of the magnetic field (in the direction of the minimum variance) can be used to obtain an estimation of $p$ (see Figure 5 (top) in Gulisano et al. [2007]), as

$$
\frac{\left\langle B_{x, \text { cloud }}\right\rangle}{B_{0}} \approx 1.6\left(\frac{p}{r}\right)^{2}+0.077\left(\frac{p}{r}\right)+0.053 .
$$

In addition, we compare the spatial extent of each substructure region relative to the size of its parent magnetic cloud and look for similarities between the overall sizes and durations of the magnetic clouds. We also calculate the average magnetic field strength over the duration of each magnetic cloud and compare the values obtained for each event.

[21] In situ observations of the velocity profiles of magnetic clouds show that they typically expand as they propagate through the heliosphere, with the decrease in total solar wind pressure with solar distance playing a significant role in driving this expansion [Démoulin and Dasso, 2009]. Magnetic clouds often exhibit a linear velocity profile, and by considering $\Delta V_{x}$ over the duration of each cloud, we calculate the nondimensional expansion rate, $\zeta$, with respect to the cloud center for each of these clouds. By observing 


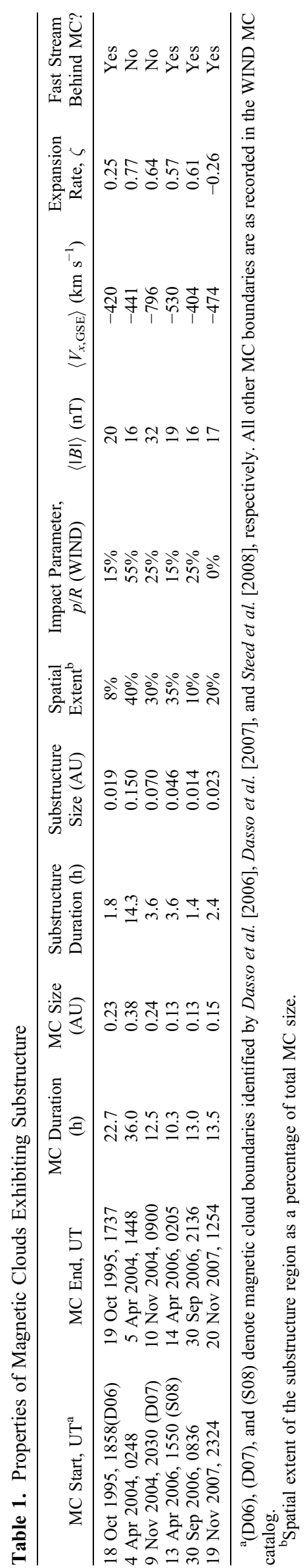

the solar wind behind each magnetic cloud, we also determine whether the cloud was being followed, and ultimately overtaken, by a solar wind stream propagating faster than the ejecta.

[22] Table 1 shows that the size and duration of each magnetic cloud varies significantly between events and that the same can be said of the size and duration of the substructure regions, both between the events and relative to the size of their parent magnetic clouds. We also find that there are no clear similarities between the trajectories of the WIND spacecraft through each of these magnetic clouds, determined from the estimated impact parameter of the spacecraft in each case, with some clouds observed closer to their axes than others. Calculations of the average magnetic field strength over the duration of each cloud reveal that it is comparable $(\sim 17 \mathrm{nT})$ in all but one of these events $(\sim 32 \mathrm{nT})$, and the values obtained are within the range expected for a typical magnetic cloud [Lepping et al., 2003]. We find that $\zeta$ is smaller than the mean value obtained for the nonperturbed (not overtaken) magnetic clouds analyzed by Démoulin et al. [2008] at $1 \mathrm{AU}(\zeta=0.8 \pm 0.2)$, and those analyzed by Gulisano et al. [2010] between 0.3 and $1 \mathrm{AU}$ $(\zeta=0.9 \pm 0.2)$. This is consistent with the presence of an overtaking solar wind stream behind these magnetic clouds, as the lowest values of $\zeta$ are found for overtaken clouds (see Table 1). There is also one case where $\zeta$ is negative, implying that when this cloud is observed by WIND it is actually compressing rather than expanding.

\section{Discussion}

[23] In this study we have investigated the properties of a subset of magnetic clouds within which substructure, in the form of multiple reversals of the gradient of the azimuthal magnetic field, is observed toward the center of the magnetic flux rope structure. A particularly clear example of substructure in a magnetic cloud was observed in situ by the WIND spacecraft on 13 April 2006, and we show that substructure is evident in both the magnetic field observations and the corresponding plasma observations. In addition to the reversals of the azimuthal magnetic field gradient and the depression of the axial magnetic field, several thin current sheets are identified within the substructure region.

[24] One of the most recent explanations for the presence of current sheets within magnetic clouds is a scenario proposed by Owens [2009], who demonstrated how current sheets can form simply as a result of a magnetic cloud's kinematic propagation from the Sun to the Earth. This model of a kinematically distorted flux rope was able to reproduce an observed discontinuity present in the magnetic structure of some magnetic clouds and demonstrate that these current sheets thin, and thus increase in intensity, as the angular width of the flux rope increases and the radial expansion speed decreases. We have shown that the rate of radial expansion of the 13 April 2006 magnetic cloud is small at $1 \mathrm{AU}$. However, unlike the examples studied by Owens [2009], the angular width of this magnetic cloud is believed to be quite small, because despite originating close to disk center, this CME is not observed by the Large Angle Spectroscopic Coronagraph (LASCO) [Brueckner et al., 1995] onboard the SoHO spacecraft [Steed et al., 2008]. Owens [2009] also hypothesise that magnetic reconnection 
occurring across such current sheets in magnetic clouds may result in a single flux rope being fragmented into multiple, smaller flux ropes.

[25] The idea of multiple flux ropes giving rise to observations of unusual ICME signatures is not a new one [e.g., Osherovich et al., 1999]. Wang et al. [2002], and many of the scenarios proposed in recent years, do not require magnetic reconnection as a driving force. For example, it has been suggested that some events observed near Earth are a result of CME-CME interaction, and Gopalswamy et al. [2001] described a scenario where a faster CME ploughs into a slower CME travelling ahead of it. In some cases, both MCs even travel consecutively and the flux rope signatures are preserved [Dasso et al., 2009]. On the other hand, Dasso et al. [2007] also considered whether the substructure could be explained by a small twisted flux tube with opposite helicity sign in the center of the flux rope and concluded that the formation of such a structure is not possible in the corona. It is, however, possible for two flux ropes with the same sign of helicity to interact in interplanetary space, but the symmetrical nature of the 13 April 2006 flux rope signature in $\mathrm{B}_{y \text {,cloud implies that the flux }}$ ropes would have to be very similar to reproduce the observed substructure signatures, and it is very improbable that this is the case.

[26] Dasso et al. [2007] also suggested the simplest interpretation of the observed substructure may be spatial oscillations of the magnetic field, introduced as a result of interactions between a magnetic cloud and the ambient solar wind as the cloud propagates into interplanetary space. They proposed that close to the minimum approach of the magnetic cloud center, the spacecraft trajectory would be nearly tangential to the magnetic flux surfaces of the flux rope. Here, the azimuthal magnetic field tends to zero and any warping of the flux surfaces would give a clear signal in the $B_{y, \text { cloud }}$ component. This could potentially give rise to a signature within the magnetic field of a flux rope that resembles the observations of substructure in $B_{y, \text { cloud }}$ identified in this study, but does not explain the observed decrease in $B_{z \text {,cloud }}$ toward the center of the flux rope. However, there are several events in this study for which "warping" is a possible explanation for the observed signatures, since a depression of $B_{z \text {,cloud }}$ is not common to all of these events, unlike the multiple reversals of the gradient of $B_{y \text {,cloud. }}$

[27] It is also possible that MHD waves may be perturbing the magnetic field on the flanks of the flux rope. For example, the Kelvin-Helmholtz instability [Dungey, 1955; Drazin and Reid, 1985] may be invoked when two fluids are in motion with respect to one another. In this case, the stability of the interface between the magnetic cloud and the solar wind is dependent on the magnitude of the velocity shear. The fast flowing plasma in the magnetic cloud moving past slower solar wind plasma may create the neccessary velocity shear across the boundary between them to invoke the instability. However, if this were the case it is not obvious how these boundary waves would result in the internal substructure signatures we observe.

[28] Similar observations of the magnetic field from the ACE and WIND spacecraft suggest that the 13 April 2006 magnetic structure does not undergo significant temporal or spatial evolution as the magnetic cloud propagates from
ACE to WIND. This may be because the magnetic cloud is evolving more slowly than we are able to detect. The close proximity of the ACE and WIND spacecraft to one another results in a timescale of only a few minutes between the respective observations, which is very small compared to typical timescales of magnetic cloud evolution, usually of the order of hours. We estimate the trajectories of the ACE and WIND spacecraft through this magnetic cloud by considering the impact parameters of the spacecraft determined from flux rope modeling, as before. We find that the values obtained for the impact parameter are very similar, as expected given the relative spacecraft positions, with a value of $p / R$ of approximately $15 \%$ for both ACE and WIND, where $R$ is the radius of the magnetic cloud.

[29] We are unable to determine from the available observations of this event when the substructure develops, but it is possible that substructure is present, or introduced, as the CME erupts. However, it is also plausible that substructure forms within an ICME as it propagates through the heliosphere. Further study of the solar sources of these types of ejecta would assist with narrowing down the point at which substructure begins to develop within an ICME.

[30] In addition to the 13 April 2006 magnetic cloud, we have identified and studied five other magnetic cloud events within which substructure is observed. We initially surveyed 48 magnetic clouds and have found that around $60 \%$ of the clouds in the WIND magnetic cloud catalog do not rotate entirely smoothly, exhibiting varying degrees of internal fluctuations of the magnetic field. However, only a very small proportion of these exhibit clear substructure signatures. These findings suggest that substructure events are rare, but we must also consider that the boundaries of the magnetic clouds included in this study, particularly those determined from the WIND magnetic cloud catalog, may not be entirely accurate. Much progress has been made in recent years in identifying the boundaries of solar ejecta by applying new methods and techniques, in making quantitative comparisons with their solar sources [e.g., Démoulin, 2008] and in using MHD invariants in space [e.g., Dasso, 2009]. Accurate identification of magnetic cloud boundaries is particularly difficult for complex events, like those in which we identify substructure.

[31] There has been much discussion and speculation about whether all ICMEs in fact contain flux ropes. However, the presence of a flux rope may not be evident in the in situ observations of many events due to the limitations of single spacecraft measurements, and particularly the trajectory of the spacecraft through the ejecta [e.g., Marubashi, 2000]. Similarly, we suggest that the same observational constraints, in particular the lack of availability of multispacecraft measurements for each event, mean that we cannot rule out the possibility that substructure may be present in more magnetic clouds than we are able to identify with the available in situ observations. All of the magnetic clouds associated with substructure identified in this study were observed during the late declining phase of the solar activity cycle, but given the small sample size of magnetic clouds, there is not yet sufficient evidence to determine if there is any dependence of the formation of substructure within MCs on the phase of the solar cycle.

[32] A comparison of the physical parameters of these magnetic clouds has revealed that they have little in common. 
Since the solar wind pressure decreases with increasing heliocentric distance, MCs typically expand as they propagate into interplanetary space. A signature of this expansion is observed in the plasma bulk flow velocity in the SunEarth direction, which is expected to decrease approximately linearly between the leading and trailing boundaries of the cloud. Conversely, magnetic clouds may become perturbed as a result of their interaction with the solar wind. In a perturbed cloud, the majority of the velocity profile of a cloud is nonlinear. Nearly all of the clouds in this study are followed by fast solar wind streams. We have found that the nondimensional expansion rates of these MCs are low, with values of $\zeta<0.8$, indicating that these magnetic clouds are perturbed [Gulisano et al., 2010]. As a fast stream overtakes a magnetic cloud, it is expected to compress the flux rope and so decrease its expansion rate, as shown in MHD simulations [Xiong et al., 2006] and in in situ observations [Gulisano et al., 2010].

\section{Conclusions}

[33] Substructure is observed within some magnetic clouds, identified by multiple reversals of the gradient of the azimuthal magnetic field of the magnetic flux rope structure. One such event was observed by the ACE and WIND spacecraft, located upstream of Earth, on 13 April 2006. We have shown that substructure is evident in both the azimuthal and axial magnetic field components and the associated plasma observations. In total, we have identified six magnetic clouds clearly exhibiting such substructure signatures, and a comparison of the properties of these clouds has revealed that they vary significantly from event to event. However, these clouds all exhibit low nondimensional expansion rates, suggesting that they are perturbed by the surrounding solar wind conditions, and fast solar wind streams are observed overtaking four of the six events studied.

[34] It is not yet understood when and where substructure develops within magnetic clouds. Further study is neccessary to investigate whether substructure is present during CME initiation and/or eruption, or if it develops as a result of the evolution of the magnetic structure as it propagates into the heliosphere. Understanding the driving forces behind the topological changes in a magnetic flux rope that give rise to substructure will be the subject of a future study, where we will explore possible scenarios that might lead to the formation of substructure within a magnetic cloud.

[35] Acknowledgments. We acknowledge the use of solar wind data from the on-line CDAW web site of NASA/GSFC. The ACE magnetic field and plasma data are courtesy of N. Ness and D. J. McComas respectively. The WIND magnetic field and plasma data are courtesy of R. Lepping and R. Lin, respectively. K. Steed thanks STFC for support via Ph.D. studentship. S. Dasso is a member of CIC (CONICET) and partially supported by grants UBACyT X425, PIP-2009-00825 (CONICET), PICT 2007-00856 (ANPCyT). P. Démoulin and S. Dasso acknowledge financial support from ECOS-Sud (France) and MINCyT (Argentina) through their cooperative science program (A08U01).

[36] Philippa Browning thanks Eberhard Moebius and another reviewer for their assistance in evaluating this paper.

\section{References}

Brueckner, G. E., et al. (1995), The Large Angle Spectroscopic Coronagraph (LASCO), Sol. Phys., 162, 357-402, doi:10.1007/BF00733434.
Burlaga, L. F. (1995), Interplanetary Magnetohydrodynamics, pp. 89-114, Oxford Univ. Press, New York.

Burlaga, L. F., E. Sittler, F. Mariani, and R. Schwenn (1981), Magnetic loop behind an interplanetary shock: Voyager, Helios and IMP 8 observations, J. Geophys. Res., 86, 6673-6684, doi:10.1029/JA086iA08p06673.

Crooker, N. U., J. T. Gosling, E. J. Smith, and C. T. Russell (1990), A bubblelike coronal mass ejection in the solar wind, in Physics of Magnetic Flux Ropes, Geophys. Monogr. Ser., vol. 58, edited by C. T. Russell, E. R. Priest, and L. C. Lees, pp. 365-371, AGU, Washington, D. C.

Dasso, S. (2009), Magnetic helicity content in solar wind flux ropes, Proc. Int. Astron. Union, 257, 379-389, doi:10.1017/S1743921309029603.

Dasso, S., C. H. Mandrini, P. Démoulin, M. L. Luoni, and A. M. Gulisano (2005), Large scale MHD properties of interplanetary magnetic clouds, Adv. Space Res., 35, 711-724, doi:10.1016/j.asr.2005.02.096.

Dasso, S., C. H. Mandrini, P. Démoulin, and M. L. Luoni (2006), A new modelindependent method to compute magnetic helicity in magnetic clouds, Astron. Astrophys., 455, 349-359, doi:10.1051/0004-6361:20064806.

Dasso, S., M. S. Nakwacki, P. Démoulin, and C. H. Mandrini (2007), Progressive transformation of a flux rope to an ICME, Sol. Phys., 244, 115-137, doi:10.1007/s11207-007-9034-2.

Dasso, S., et al. (2009), Linking two consecutive nonmerging magnetic clouds with their solar sources, J. Geophys. Res., 114, A02109, doi:10.1029/2008JA013102.

Démoulin, P. (2008), A review of the quantitative links between CMEs and magnetic clouds, Ann. Geophys., 26, 3113-3125.

Démoulin, P., and S. Dasso (2009), Causes and consequences of magnetic cloud expansion, Astron. Astrophys., 498, 551-566, doi:10.1051/0004$6361 / 200810971$

Démoulin, P., M. S. Nakwacki, S. Dasso, and C. H. Mandrini (2008), Expected in situ velocities from a hierarchical model for expanding interplanetary coronal mass ejections, Sol. Phys., 250, 347-374, doi:10.1007/ s11207-008-9221-9.

Drazin, P. G., and W. H. Reid (1985), Hydromagnetic Instability, Cambridge Univ. Press, New York

Dungey, J. W. (1955), Electrodynamics of the outer atmosphere, in Proceedings of the Ionosphere Conference, p. 225, Phys. Soc. of London, London.

Fainberg, J., V. A. Osherovich, R. G. Stone, R. J. MacDowell, and A. Balogh (1996), Ulysses observations of electron and proton components in a magnetic cloud and related wave activity, in Solar Wind Eight, edited by J. T. Gosling et al., AIP. Conf. Proc., 382, 554-557, doi:10.1063/ 1.51513 .

Farrugia, C. J. (2001), A reconnection layer associated with a magnetic cloud, Adv. Space Res., 28, 759-764, doi:10.1016/S0273-1177(01) 00529-4.

Gopalswamy, N., S. Yashiro, M. L. Kaiser, R. A. Howard, and J. L. Bougeret (2001), Radio signatures of coronal mass ejection interaction: Coronal mass ejection cannibalism?, Astrophys. J., 548, L91-L94, doi:10.1086/ 318939.

Gosling, J. T. (1990), Coronal mass ejections and magnetic flux ropes in interplanetary space, in Physics of Magnetic Flux Ropes, Geophys. Monogr. Ser., vol. 58, edited by C. T. Russell, E. R. Priest, and L. C. Lees, pp. 343-364, AGU, Washington, D. C.

Gosling, J. T., D. N. Baker, S. J. Bame, W. C. Feldman, R. D. Zwickl, and E. J. Smith (1987), Bidirectional solar wind electron heat flux events, J. Geophys. Res., 92, 8519-8535, doi:10.1029/JA092iA08p08519.

Gosling, J. T., S. Eriksson, D. J. McComas, T. D. Phan, and R. M. Skoug (2007), Multiple magnetic reconnection sites associated with a coronal mass ejection in the solar wind, J. Geophys. Res., 112, A08106, doi:10.1029/2007JA012418.

Gulisano, A. M., S. Dasso, C. H. Mandrini, and P. Démoulin (2007), Estimation of the bias of the minimum variance technique in the determination of magnetic clouds global quantities and orientation, Adv. Space Res., 40, 1881-1890, doi:10.1016/j.asr.2007.09.001.

Gulisano, A. M., P. Démoulin, S. Dasso, M. E. Ruiz, and E. Marsch (2010), Global and local expansion of magnetic clouds in the inner heliosphere, Astron. Astrophys., 509, A39, doi:10.1051/0004-6361/200912375.

Hirshberg, J., S. J. Bame, and D. E. Robbins (1972), Solar flares and solar wind helium enrichments: July 1965-July 1967, Sol. Phys., 23, 467-486, doi:10.1007/BF00148109.

Klein, L. W., and L. F. Burlaga (1982), Interplanetary magnetic clouds at 1 AU, J. Geophys. Res., 87, 613-624, doi:10.1029/JA087iA02p00613.

Lepping, R. P., et al. (1995), The WIND magnetic field investigation, Space Sci. Rev., 71, 207-229, doi:10.1007/BF00751330.

Lepping, R. P., et al. (1997), The Wind magnetic cloud and events of October 18-20, 1995: Interplanetary properties and as triggers for geomagnetic activity, J. Geophys. Res., 102, 14,049-14,063, doi:10.1029/97JA00272.

Lepping, R. P., D. B. Berdichevsky, A. Szabo, C. Arqueros, and A. J. Lazarus (2003), Profile of an average magnetic cloud at $1 \mathrm{AU}$ for the 
quiet solar phase: Wind observations, Sol. Phys., 212, 425-444, doi:10.1023/A:1022938903870.

Lepping, R. P., J. A. Jones, and L. F. Burlaga (1990), Magnetic field structure of interplanetary magnetic clouds at $1 \mathrm{AU}, J$. Geophys. Res., 95, 11,957-11,965, doi:10.1029/JA095iA08p11957.

Lin, R. P., et al. (1995), A three-dimensional plasma and energetic particle investigation for the wind spacecraft, Space Sci. Rev., 71, 125-153, doi:10.1007/BF00751328.

Lopez, R. E. (1987), Solar cycle invariance in solar wind proton temperature relationships, J. Geophys. Res., 92, 11,189-11,194, doi:10.1029/ JA092iA10p11189.

Lundquist, S. (1950), Magnetostatic fields, Ark. Fys., 2, 361-365.

Lynch, B. J., T. H. Zurbuchen, L. A. Fisk, and S. K. Antiochos (2003), Internal structure of magnetic clouds: Plasma and composition, J. Geophys. Res., 108(A6), 1239, doi:10.1029/2002JA009591.

Marubashi, K. (1997), Interplanetary magnetic flux ropes and solar filaments, in Coronal Mass Ejections, Geophys. Monogr. Ser., vol. 99 edited by N. Crooker, J. Jocelyn, and J. Feynman, pp. 147-156, AGU, Washington, D. C

Marubashi, K. (2000), Physics of interplanetary flux ropes: Toward prediction of geomagnetic storms, Adv. Space Res., 26, 55-66, doi:10.1016/ S0273-1177(99)01026-1.

McComas, D. J., S. J. Bame, P. Barker, W. C. Feldman, J. L. Phillips, P. Riley, and J. W. Griffee (1998), Solar Wind Electron Proton Alpha Monitor (SWEPAM) for the Advanced Composition Explorer, Space Sci. Rev., 86, 563-612, doi:10.1023/A:1005040232597.

Mulligan, T., C. T. Russell, and J. T. Gosling (1999), On interplanetary coronal mass ejection identification at $1 \mathrm{AU}$, in Solar Wind Nine, edited by S. R. Habbal et al., AIP Conf. Proc., 471, 693-696.

Neugebauer, M., J. T. Steinberg, R. L. Tokar, B. L. Barraclough, E. E. Dors, R. C. Wiens, G. E. Gingerich, D. Luckey, and D. B. Whiteaker (2003), Genesis on-board determination of the solar wind flow regime, Space Sci. Rev., 105, 661-679, doi:10.1023/A:1024478129261.

Osherovich, V. A., J. Fainberg, and R. G. Stone (1999), Multi-tube model for interplanetary magnetic clouds, Geophys. Res. Lett., 26, 401-404, doi:10.1029/1998GL900306.

Owens, M. J. (2009), The formation of large-scale current sheets within magnetic clouds, Sol. Phys., 260, 207-217, doi:10.1007/s11207-0099442-6.

Richardson, I. G., and H. V. Cane (1995), Regions of abnormally low proton temperature in the solar wind (1965-1991) and their association with ejecta, J. Geophys. Res., 100, 23,397-23,412, doi:10.1029/95JA02684.
Richardson, I. G., and H. V. Cane (2004), Identification of interplanetary coronal mass ejections at $1 \mathrm{AU}$ using multiple solar wind plasma composition anomalies, J. Geophys. Res., 109, A09104, doi:10.1029/ 2004JA010598.

Richardson, I. G., D. Berdichevsky, M. D. Desch, and C. J. Farrugia (2000), Solar-cycle variation of low density solar wind during more than three solar cycles, Geophys. Res. Lett., 27, 3761-3764.

Russell, C. T., L. K. Jian, and J. G. Luhmann (2009), An unusual current sheet in an ICME: Possible association with C/2006 P1 (McNaught), Geophys. Res. Lett., 36, L07105, doi:10.1029/2009GL037615.

Siscoe, G. L., and R. W. Suey (1972), Significance criteria for variance matrix applications, J. Geophys. Res., 77, 1321-1322, doi:10.1029/ JA077i007p01321.

Sonnerup, B. U., and J. L. Cahill (1967), Magnetosphere structure and attitude from Explorer 12 observations, J. Geophys. Res., 72, 171-183, doi:10.1029/JZ072i001p00171.

Steed, K., C. J. Owen, L. K. Harra, L. M. Green, S. Dasso, A. P. Walsh, P. Démoulin, and L. van Driel-Gesztelyi (2008), Locating the solar source of 13 April 2006 magnetic cloud, Ann. Geophys., 26, 3159-3168.

Wang, Y. M., S. Wang, and P. Z. Ye (2002), Multiple magnetic clouds in interplanetary space, Sol. Phys., 211, 333-344, doi:10.1023/ A: 1022404425398

Xiong, M., H. Zheng, Y. Wang, and S. Wang (2006), Magnetohydrodynamic simulation of the interaction between interplanetary strong shock and magnetic cloud and its consequent geoeffectiveness, J. Geophys. Res., 111, A08105, doi:10.1029/2005JA011593.

Zwickl, R. D., J. R. Asbridge, S. J. Bame, W. C. Feldman, J. T. Gosling, and E. J. Smith (1983), Plasma properties of driver gas following interplanetary shocks observed by ISEE-3, in Solar Wind Five, edited by M. Neugebauer, NASA Conf. Proc., 2280, 711-717.

S. Dasso, Departmento de Fisica, CONICET-UBA, CC. 67, Suc. 28 , 1428 Buenos Aires, Argentina.

P. Démoulin, Observatoire de Paris, LESIA, UMR 8109 (CNRS), F-92195 Meudon, France.

C. J. Owen and K. Steed, Mullard Space Science Laboratory, University College London, Holmbury St. Mary, Dorking, Surrey RH5 6NT, United Kingdom. (ks3@mssl.ucl.ac.uk) 